\title{
A TDL Based Non-WSSUS Vehicle-to-Vehicle Channel Model
}

\author{
Yan Li, ${ }^{1}$ Bo Ai, ${ }^{1}$ Xiang Cheng, ${ }^{2}$ Siyu Lin, ${ }^{3}$ and Zhangdui Zhong' \\ ${ }^{1}$ State Key Laboratory of Rail Traffic Control and Safety, Beijing Jiaotong University, No. 3 Shang Yuan Cun, Haidian District, \\ Beijing 100044, China \\ ${ }^{2}$ School of Electronics Engineering and Computer Science, Peking University, No. 5 Yiheyuan Road, Haidian District, \\ Beijing 100871, China \\ ${ }^{3}$ School of Electronic and Information Engineering, Beijing Jiaotong University, No. 3 Shang Yuan Cun, Haidian District, \\ Beijing 100044, China \\ Correspondence should be addressed to Bo Ai; aibo@ieee.org
}

Received 9 July 2013; Accepted 18 October 2013

Academic Editor: César Briso Rodríguez

Copyright (C) 2013 Yan Li et al. This is an open access article distributed under the Creative Commons Attribution License, which permits unrestricted use, distribution, and reproduction in any medium, provided the original work is properly cited.

\begin{abstract}
This paper proposes a non-wide-sense-stationary-uncorrelated scattering (WSSUS) channel model for vehicle-to-vehicle (V2V) communication systems. The proposed channel model is based on the tapped-delay line (TDL) structure and considers the correlation between taps both in amplitude and phase. Using the relationship between the correlation coefficients of complex Gaussian, Weibull, and Uniform random variables (RVs), the amplitude and the phase of taps with different delays are modeled as correlated RVs to reflect the non-WSSUS properties of V2V channels. The effectiveness of the proposed channel model and simulation method is validated by the measurements in different scenarios.
\end{abstract}

\section{Introduction}

Vehicle-to-vehicle (V2V) communication system is a promising part of Intelligent Transportation Systems (ITS) [1], which can provide various of information services, such as the accident notification and the real-time road traffic reports, to improve the safety and the efficiency of the transportation system. The $\mathrm{V} 2 \mathrm{~V}$ communication system is a type of ad hoc communication network; different cars can exchange information with each other to provide a comfort and "infotainment" environment for driver. The IEEE 802.11p is a new V2V communication standard in the $5.9 \mathrm{GHz}$ Unlicensed National Information Infrastructure (UNII) band, which is an approved amendment to the IEEE 802.11a standard by adding some extended functions to adapt to vehicle communication environment. IEEE 802.11p has been admitted as the communication standard for dedicated short range communications (DSRC) of the ITS in USA [2].

Channel modeling is the foundation for design and simulation of communication systems, whose accuracy plays a key role in optimizing and improving the performance of V2V communication systems [3]. The propagation characteristics of V2V channels in different scenarios, such as in the urban, the suburban, and the highway, are quite different from those of traditional cellular channels. The characteristics of V2V channels contain (i) both the transmitter and the receiver are moving, (ii) the significant scatterers/reflectors are also changing continuously, (iii) the omnidirectional antennas for both Tx and Rx are at low heights, and (iv) the environment changes dynamically as vehicles move. In traditional channel modeling, the wide-sense stationary uncorrelated scattering (WSSUS) assumption has been widely used to describe random linear time-varying cellular channels [4]. In V2V channels, due to the dynamically changing environment, the statistics of channel changes over moderate time scales (nonWSS); due to the several taps interacting with one-and-thesame object, such as buildings on the road side, the taps at different delays show correlated fading property (non-US). Therefore, the WSSUS assumption is not valid for accurate description of V2V radio communication channels. This specific characteristics of $\mathrm{V} 2 \mathrm{~V}$ channels are not adequately reproduced by standard TDL models, which leads us to characterize the non-WSSUS properties of V2V channels by developing a non-WSSUS channel model.

To fill the aforementioned gap, this paper proposes a new TDL based non-WSSUS V2V channel model that for the first 
time considers the amplitude and phase correlations between different taps. Using the relationship between the correlation coefficients of complex Gaussian, Weibull, and Uniform random variables (RVs), correlated taps are generated with arbitrary amplitude and phase correlation coefficients, arbitrary fading parameters, and arbitrary energies. In this model, the correlated taps represent the correlation at different delays, which is caused by the several taps interacting with one-andthe-same object. The model multiplies the switching function $z_{l}(t)=\{0,1\}$ with the first-order two-state Markov chains to represent the non-WSS properties, which is caused by the short-time averaged power associated with a reflector fluctuates and the delays of the channel taps drift as the distances between the transmitter and the receiver.

The remainder of this paper is organized as follows. In Section 2, we review the related works of V2V channel models. In Section 3, we review the TDL models and the characteristics of V2V channels. Section 4 proposes a new non-WSSUS V2V channel model, whose taps at different delays are correlated in both the amplitude and phase. Section 5 validates the accuracy of the proposed channel model and evaluates the performances of OFDM systems under the condition of $\mathrm{V} 2 \mathrm{~V}$ channels.

\section{Related Work}

There are many literatures focusing on developing accurate models for $\mathrm{V} 2 \mathrm{~V}$ channels. V2V channel models can be classified as two classes, the geometry-based deterministic models (GBDMs) and the stochastic models, while the stochastic models can be further categorized as the geometrybased stochastic models (GBSMs) and the nongeometrical stochastic models (NGSMs) [5]. The GBDMs characterize typical channel parameters in a deterministic approach. A GBDM based on ray-tracing methods for $\mathrm{V} 2 \mathrm{~V}$ systems is the pioneering work by Maurer et al. [6, 7]; this model aims at developing a site-specific and actual physical radio propagation process for a given environment. However, the model cannot easily be generalized to more scenarios [8]. The GBSMs are used to simulate the channel by predefined stochastic distribution of effective scatters around the transmitter and the receiver. The GBSMs can be further divided into the regular-shaped GBSMs and the irregular-shaped GBSMs. The simplest regular-shaped GBSM is the two-ring model, which is proposed by Akki and Haber [9]. The tworing and ellipse model constructs the received signal as a sum of the line-of-sight, single, and double bounced rays with different energies [10]. The classic two-ring model assumes that all scatters are placed in the horizontal plane, while in reality the scatters and reflectors are in different heights. Then the two-ring model is extended to the three-dimensional model, which places the scatters in the two-concentric cylinder around the transmitter and the receiver [11]. In the irregular-shaped GBSMs model, mobile discrete scatters, static discrete scatters, and diffuse scatters are randomly generated [12]. Due to the static nature of the geometry, the GBSMs can generalize to more scenarios; however, the model cannot represent the non-WSSUS properties of the
$\mathrm{V} 2 \mathrm{~V}$ channels. NGSMs determine the model parameters in a completely statistic manner with a certain delay and Doppler shift. In NGSMs, the impulse response of the V2V channel is modeled with taps at certain delays; hence the channel is modeled based on the tapped-delay line (TDL) structure [4]. The sum-of-sinusoids (SoS) simulation method can be used to simulate the V2V channel models [13]. Furthermore, the wideband channel model and channel statistics are discussed in $[14,15]$. Among all kinds of channel models, NGSMs are widely used to mimic non-WSSUS for V2V channels due to its low complexity and acceptable accuracy.

IEEE 802.11p employs the 6-tap and 12-tap NGSMs [16, 17] as basic channel model; each tap consists of several paths, and each path has different types of Doppler spectrum (flat shape, round shape, classic $3 \mathrm{~dB}$ shape, and classic $6 \mathrm{~dB}$ shape). This allows almost arbitrary Doppler spectrum to be synthesized for each tap. However, these models are based on the WSSUS assumption, since they assume that the different taps in TDL structure are independent and uncorrelated to each other. David and coworkers describe the non-WSS property of channel by modeling multipath component persistence via Markov chains [18]. Over moderate time scales, the scatters and reflectors will be "Death and Birth" caused by dynamic blockage or obstacles nearby; the model describes the nonWSS phenomenon by random on/off process. Furthermore, the model generates multivariate Weibull variables with arbitrary fading parameters, arbitrary average fading powers, and arbitrary amplitude correlations to represent the nonWSSUS properties, while none of the above models considers the complex correlations between different taps, including both the amplitude correlations and the phase correlations. To accurately describe the channel characteristics of $\mathrm{V} 2 \mathrm{~V}$ communication systems, the V2V channel should be modeled as non-WSSUS process.

\section{Channel Models}

In this section, we first review the traditional TDL based models and discuss the non-WSS and non-US properties of $\mathrm{V} 2 \mathrm{~V}$ channels. Then the TDL based V2V channel model is proposed to represent the non-WSSUS properties.

3.1. TDL Based Models. The TDL based models are originated from the assumption that the resolvable taps of channels can be approximated as discrete taps with different delays [4]. The models are used to represent the WSSUS linear randomly time-varying channel. The channel impulse response (CIR) of the TDL models can describe completely the channel characteristics, which can be denoted as

$$
\begin{aligned}
h(\tau, t)= & \sum_{l=0}^{L(t)-1} \alpha_{l}(t) \delta\left[\tau-\tau_{l}(t)\right] \\
& \times \exp \left\{j\left[\omega_{D, l}(t)\left(t-\tau_{l}(t)\right)-\omega_{c}(t) \tau_{l}(t)\right]\right\} \\
= & \sum_{l=0}^{L(t)-1} g_{l}(t) \delta\left[\tau-\tau_{l}(t)\right]
\end{aligned}
$$


where $l$ represents the index of taps, $\alpha_{l}(t)$ represents the $l$ th $(l=1, \ldots, L)$ resolved amplitude, the exponential term represents the phase of the lth tap, and $\tau_{l}(t)$ represents the express delay of the $l$ th tap. $\omega_{c}=2 \pi f_{c}$ is the radian carrier frequency; $\omega_{D, l}(t)=2 \pi f_{D, l}(t)$, where $f_{D, i}(t)=$ $v(t) f_{c} \cos \left[\theta_{l}(t)\right] / c$ is the Doppler frequency induced by the motion of the Tx and $\mathrm{Rx}, v(t)$ denotes the relative velocity, $\theta_{l}(t)$ is the aggregate phase angle of all components arriving in the $l$ th tap, and $c$ is the speed of light. The TDL model is illustrated in Figure 1.

In $\mathrm{V} 2 \mathrm{~V}$ channels, due to the dynamic environment and large obstacles, the channels are non-WSSUS. Traditional TDL models assume that the taps are independent, while this assumption cannot be hold in $\mathrm{V} 2 \mathrm{~V}$ channels.

3.2. Non-WSSUS Model. In V2V channels, the transmitter, receiver, reflectors, and scatters around are mobile, so the propagation environment changes dynamically. The significant characteristics of V2V channels are non-WSS and nonUS. Non-WSS property describes the channel stochastic characteristics changed with time scale, which represents the time domain characteristics. While non-US property reflects the impacts of correlation on different paths, which represents the delay domain characteristics. In V2V channels, the scatterers and the reflectors will be "Death and Birth" over moderate time scales, which can be modeled by the multipath component persistence as a random on/off process. It can be described as the first-order two-state Markov chains [18]

$$
\mathbf{T S}=\left[\begin{array}{ll}
p_{00} & p_{01} \\
p_{10} & p_{11}
\end{array}\right], \quad \mathbf{S S}=\left[\begin{array}{l}
p_{0} \\
p_{1}
\end{array}\right],
$$

where TS is the state transition matrix, $p_{u v}$ is the transition probability of transition from state $u$ to state $v$, the state 0 represents the existence of taps, and state 1 represents the disappearance of taps. The SS represents the steady-state matrix and $p_{u}$ represents the steady-state probability of state $u$, which satisfies $p_{00}+p_{01}=1, p_{10}+p_{11}=1$, and $p_{0}+p_{1}=1$.

The state decision interval of "Death and Birth" state is defined as channel coherence time $T_{c}=9 / 16 \pi f_{m}$, where $f_{m}$ is the maximum Doppler frequency. Compared to traditional TDL models, a switching function $z_{l}(t)=\{0,1\}$ is proposed to reflect the non-WSS property.

In $\mathrm{V} 2 \mathrm{~V}$ channels, the taps at different delays are correlated, due to the several taps interacting with one-and-the same object, such as buildings on the road side. As shown in Figure 2, the correlated complex Gaussian RVs can be generated with any desired correlation coefficients.

The process to generate correlated complex Gaussian RVs with correlation $\rho^{G}$ from pairs of uncorrelated Gaussian RVs is as follows. (i) Generate uncorrelated complex Gaussian RVs, which are denoted as $V$. (ii) Determine the coloring matrix $L$ by Cholesky decomposition of the correlation matrix $L L^{H}=\rho^{G}$ [19], where $L^{H}$ is the Hermitian transpose of $L$. (iii) Generate correlated complex Gaussian RVs using $W=L V$. The channel correlation property can be realized with correlated taps.

According to the wireless propagation schemes between the transmitter and the receiver in $\mathrm{V} 2 \mathrm{~V}$ channels, the receivers receive the signal primarily from a particular direction

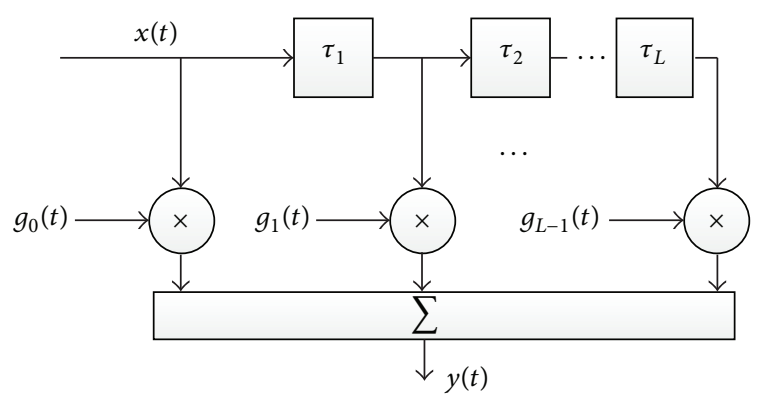

Figure 1: TDL based models.

through a narrow beamwidth. On the other hand, the scatters around may lead to different impacts of signal propagation toward the receiver. In the general environment of nonisotropic scattering, which corresponds to directional signal reception, the receivers receive the signal only from particular directions [20]. The uniform distribution is not suitable for the angle distribution of the taps in V2V channels, while the Laplace distribution is suitable for the description of the signal from a particular direction to the receiver. In this paper, the Laplace distribution is used to represent the angle distribution, which is an extension of the classic Uniform distribution model

$$
f(x \mid \mu, b)=\frac{1}{2 b} \exp \left(-\frac{|x-\mu|}{b}\right),
$$

where $\mu$ is the location parameter and $b \geq 0$ which is the scale parameter referred to as the diversity.

In V2V channels, the Weibull distribution is a better fit over all empirical data than the Nakagami distribution [18]. The taps amplitude statistics can be modeled as the flexible Weibull distribution

$$
f_{W}(\omega)=\frac{\beta}{\Omega} \omega^{\beta-1} \exp \left(\frac{-\omega^{-\beta}}{\Omega}\right),
$$

where $\beta$ is the fading parameter. When $\beta=2$, the Weibull distribution can be transformed to the Rayleigh distribution. With $\beta$ being increased, the channel quality becomes better; when $\beta$ is large enough, it means that there exits line-of-sight components. The average power is given by

$$
E\left[W^{2}\right]=\Omega^{2 / \beta} \Gamma\left[1+\left(\frac{2}{\beta}\right)\right],
$$

where $E(\cdot)$ is the expected value, $\Gamma(\cdot)$ is the gamma function, and $\Omega=E\left(\omega^{\beta}\right)$ is the power parameter. Rayleigh distribution can be transformed to Weibull distribution through the variable transformation $W=R^{2 / \beta}$.

3.3. Proposed Channel Model. In this section, a TDL based non-WSSUS V2V channel model is proposed. The channel model is defined as CIR $h(\tau, t)=\sum_{l=0}^{L(t)-1} z_{l}(t) \alpha_{l}(t) e^{j \theta_{l}(t)}$, where the amplitude $\alpha_{l}(t)$ follows the Weibull distribution, the phase $\theta_{l}(t)$ follows the Laplace distribution, and $z_{l}(t)$ is the switching function. As shown in Figure 3, the model is 


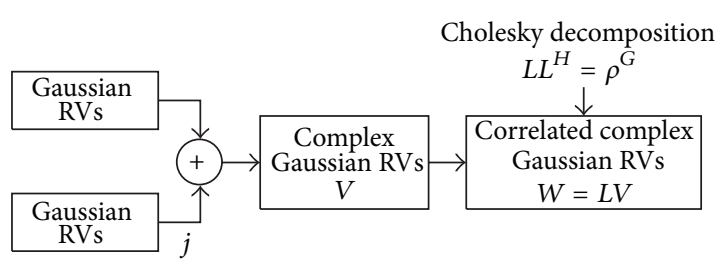

FIGURE 2: Generating correlated complex gaussian RVs.

used to generate correlated taps with arbitrary amplitude and phase correlation coefficients, fading parameters, and energy parameters.

The complex Gaussian correlation coefficients $\rho_{m, n}^{(Y)}$ can be derived with the desired Weibull correlation coefficients $\rho_{m, n}^{(W)}$, Uniform correlation coefficients $\rho_{m, n}^{(U)}$, and energy parameters $\Omega_{m}$. Define $Y=a e^{j \theta}$ as complex Gaussian RVs, where the amplitude $a$ follows Rayleigh distribution, and the phase $\theta$ follows Uniform distribution. The element $\rho_{m, n}^{(Y)}$ is the $m$ th row and $n$th column of complex Gaussian correlation coefficients matrix $\rho^{(Y)}$, which is defined as

$$
\rho_{m, n}^{(Y)}=\frac{E\left[Y_{m} Y_{n}\right]-E\left[Y_{m}\right] E\left[Y_{n}\right]}{\sqrt{\operatorname{var}\left[Y_{m}\right] \operatorname{var}\left[Y_{n}\right]}},
$$

where $Y_{m}$ and $Y_{n}$ are complex Gaussian RVs and $\operatorname{var}(\cdot)$ denotes variance.

Since the complex random variables are generated from the independent Gaussian random variables, we can prove that the amplitude and phase of the complex random variables are independent on each other; then the first term of the numerator in (6) is given by

$$
E\left[Y_{m} Y_{n}\right]=E\left[a_{m} a_{n}\right] E\left[e^{j \theta_{m}} e^{j \theta_{n}}\right]
$$

The Rayleigh RVs are $a_{m}$ and $a_{n}$, respectively; using the transformation $R=W^{\beta / 2}$ between Rayleigh RVs and Weibull RVs, the expected value $E\left[a_{m} a_{n}\right]$ is

$$
E\left[a_{m} a_{n}\right]=\iint_{0}^{\infty} \omega_{m}^{\beta_{m} / 2} \omega_{n}^{\beta_{n} / 2} f_{W_{m}, W_{n}}\left(\omega_{m}, \omega_{n}\right) d \omega_{m} d \omega_{n},
$$

where $f_{W_{m}, W_{n}}\left(\omega_{m}, \omega_{n}\right)$ is the joint pdf of the bivatiate Weibull distribution, which is given by [21] the following:

$$
\begin{aligned}
f_{W_{m}, W_{n}}\left(\omega_{m}, \omega_{n}\right) & \\
= & \frac{\beta_{m} \beta_{n} \omega_{m}^{\beta_{m}-1} \omega_{n}^{\beta_{n}-1}}{\Omega_{m} \Omega_{n}\left(1-\rho_{m, n}^{(W)}\right)} \exp \left[-\frac{1}{\left(1-\rho_{m, n}^{(W)}\right)} \times\left(\frac{\omega_{m}^{\beta_{n}}}{\Omega_{m}}+\frac{\omega_{n}^{\beta_{n}}}{\Omega_{n}}\right)\right] \\
& \times I_{0}\left[\frac{2 \sqrt{\rho_{m, n}^{(W)}} \omega_{m}^{\beta_{m} / 2} \omega_{n}^{\beta_{n} / 2}}{\left(1-\rho_{m, n}^{(W)}\right) \sqrt{\Omega_{m} \Omega_{n}}}\right],
\end{aligned}
$$

where $I_{0}(x)$ is the zero-order modified Bessel function of the first kind. This joint bivariate Weibull distribution assumes

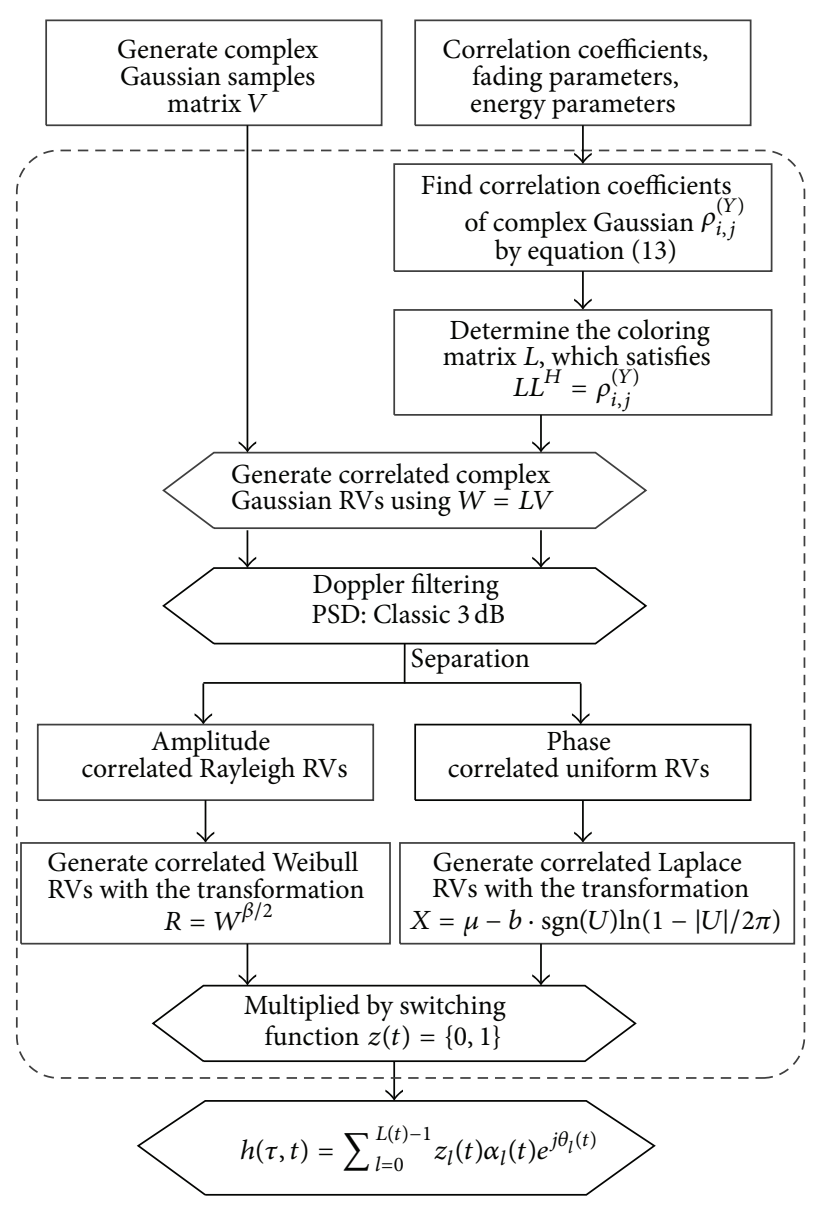

FIGURE 3: A TDL based non-WSSUS channel model.

that the correlation coefficients of the Rayleigh and Weibull RVs are identical. Even for $\beta_{m}=\beta_{n}=2$, the joint pdf can be given by (9).

The Uniform distribution RVs are $\theta_{m}$ and $\theta_{n}$; then the second term of (7) is given as follows:

$$
E\left[e^{j \theta_{m}} e^{j \theta_{n}}\right]=\iint_{0}^{2 \pi} e^{j \theta_{m}} e^{j \theta_{n}} f_{U_{m}, U_{n}}\left(\theta_{m}, \theta_{n}\right) d \theta_{m} d \theta_{n}
$$

To calculate the joint distribution, a general family of bivariate joint distribution is proposed by Morgenstern's family found in [22] with specified marginal distributions $F_{U_{m}}\left(\theta_{m}\right)$ and $F_{U_{n}}\left(\theta_{n}\right)$ as

$$
\begin{aligned}
F_{U_{m}, U_{n}}\left(\theta_{m}, \theta_{n}\right) & \\
=F_{U_{m}}\left(\theta_{m}\right) F_{U_{n}}\left(\theta_{n}\right)[ & 1+\alpha\left(1-F_{U_{m}}\left(\theta_{m}\right)\right) \\
\times & \left.\times\left(1-F_{U_{n}}\left(\theta_{n}\right)\right)\right],
\end{aligned}
$$

where $\alpha \in[-1,1]$ is the association parameter between $U_{m}$ and $U_{n}$. Then we can derive the bivariate uniform distribution which belongs to the Morgenstern's family. 
Consider the following:

$$
f_{U_{m}, U_{n}}\left(\theta_{m}, \theta_{n}\right)=\frac{1}{4 \pi^{2}}\left[1+\alpha\left(1-\frac{\theta_{m}}{\pi}\right)\left(1-\frac{\theta_{n}}{\pi}\right)\right] \text {, }
$$

where $U_{m}$ and $U_{n}$ are uniformly distributed RVs in the interval $[0,2 \pi]$. The correlation coefficients $\rho$ for the bivariate uniform distribution under study are given by $\rho=\alpha / 3$; that is, the correlation coefficients cannot exceed $1 / 3$.

Substite the general expression for the joint Weibull distribution (9) into (8) to get the expectation of $E\left[a_{m} a_{n}\right]$. Use the classic expression of the joint Uniform distribution (12) into (10) to get the exponential expectation $E\left[e^{j \theta_{m}} e^{j \theta_{n}}\right]$. Then calculating (7), we can derive the element $\rho_{m, n}^{(Y)}$ of the complex Gaussian correlation coefficients matrix

$$
\begin{aligned}
\rho_{m, n}^{(Y)}= & -\frac{3}{8 \pi} \sqrt{\Omega_{m} \Omega_{n}}\left(1-\rho_{m, n}^{(W)}\right)^{2} \\
& \times \rho_{m, n}^{(U)} H\left(\left[\frac{3}{2}, \frac{3}{2}\right], 1, \rho_{m, n}^{(W)}\right),
\end{aligned}
$$

where $H(a, d, z)$ is the traditional Gaussian hypergeometric function. It is ideal to realise that the function is related to the Weibull RVs power $\Omega_{m}$ and the correlation coefficients $\rho_{m, n}^{(W)}$ and $\rho_{m, n}^{(U)}$.

As shown in Figure 2, generate correlated complex Gaussian RVs with the complex Gaussian correlation coefficients matrix $\rho^{(Y)}$ and the RVs of uncorrelated complex Gaussian $V$. The coloring matrix $L$ can be generated by the Cholesky decomposition $L L^{H}=\rho^{(Y)}$; then the correlated complex Gaussian RVs are derived by $W=L V$.

Separate the correlated complex Gaussian RVs into the amplitude parts and the phase parts, where the amplitude parts follow Rayleigh distribution and the phase parts follow Uniform distribution.

Using the convenient transformation $R=W^{\beta / 2}$ between Weibull and Rayleigh RVs, correlated Weibull RVs can be derived from the Rayleigh RVs.

The method for generating correlated Laplace RVs is to derive from the correlated Uniform RVs, because the convenient transformation from Uniform RVs to Laplace RVs. Assuming that $U$ are uniformly distributed in the interval $[0,2 \pi]$, then we can find the Laplace distribution with parameters $\mu$ and $b$ as follows:

$$
X=\mu-b \cdot \operatorname{sgn}(U) \ln \left(1-\frac{|U|}{2 \pi}\right),
$$

where $\operatorname{sgn}(\cdot)$ is Sgn function.

Multiply the switching function $z_{l}(t)=\{0,1\}$, which is decided by the first-order two-state Markov chains illustrated in (2).

Then a TDL based non-WSSUS channel model is proposed by $h(\tau, t)=\sum_{l=0}^{L(t)-1} z_{l}(t) \alpha_{l}(t) e^{j \theta(t)}$, which considers the correlation between taps both in amplitude and phase.

\section{Simulation Results and Analysis}

In this section, we validate the accuracy of the TDL based non-WSSUS V2V channel model and evaluate the performances of the OFDM system under the proposed channel model condition. Table 1 provides the channel parameters for $10 \mathrm{MHz}$ bandwidth V2V channels [18]. The measurement scenarios are classified as the urban-antenna inside car (UIC), the urban-antenna outside car (UOC), the Small City (S), the open-area low traffic density (OLT), and the open-area high traffic density (OHT), which are conducted in five cities in different time and under different traffic conditions. The sum of all tap energies multiplied by their steady-state probabilities $P_{1}$ equals to unity, and the energy is concentrated in the first tap. The Weibull shape factors represent the fading property of the Weibull distribution. For the tap persistence process, we use the method of modeling an on/off process by Markov chains. $P_{u v, K}$ is the probability of transition from the state $u$ to the state $v$, where the states $u$ and $v$ describe the existence of taps, $u=0$ means the tap is not existing, $u=1$ means the tap is existing, and $K$ is the number of the tap. $P_{1}$ represents the probability of state 1 and $P_{0}=1-P_{1}$ is the probability of state 0 . The statistics of $\mathrm{V} 2 \mathrm{~V}$ channels change over time; the taps may be disappeared by the obstacles; then we use the random on/off process by Markov chains to describe the "Death and Birth" process.

Tables 2, 3, 4, 5 and 6, describe the matrices of the tap correlation coefficients for the scenarios: UIC, UOC, Small City, OHT, and OLT. Since the matrices of the correlation coefficients are symmetric about the diagonal, the tables are simplified as follows. The upper triangular part is phase correlation coefficients, which is generated randomly. The lower triangular part is amplitude correlation coefficients, which is measured from the scenarios: UIC, UOC, Small City, OHT, and OLT.

The procedure to develop a TDL based V2V channel model which considers the non-WSSUS channel characteristics is given as follows. (i) The correlated taps are generated with the amplitude and phase correlation coefficients and average energies, which represents the correlation at different delays (non-US). (ii) The amplitude and phase are separated from complex correlated taps; then the correlated Weibull RVs can be transformed from Rayleigh RVs and the correlated Laplace RVs can be transformed from Uniform RVs. (iii) Multiply the switching function $z_{l}(t)=\{0,1\}$ with the first-order two-state Markov chains; the random on/off process represents the changing of fading process (non-WSS). The taps of the TDL based V2V channel model are correlated, where the amplitude of the generating taps follows Weibull distribution and the phase follows Laplace distribution. Then we calculate that the correlation coefficients matrix of the generated Weibull RVs is

$$
\rho_{\mathrm{Gen}}^{(W)}=\left[\begin{array}{cccc}
1 & 0.6255 & 0.6921 & 0.6303 \\
0.6255 & 1 & 0.5418 & 0.4622 \\
0.6921 & 0.5418 & 1 & 0.8130 \\
0.6303 & 0.4622 & 0.8130 & 1
\end{array}\right]
$$

and the correlation coefficients matrix of the generated Uniform RVs is

$$
\rho_{\mathrm{Gen}}^{(U)}=\left[\begin{array}{cccc}
1 & 0.2705 & 0.0428 & 0.1077 \\
0.2705 & 1 & 0.2683 & 0.1850 \\
0.0428 & 0.2683 & 1 & 0.3069 \\
0.1077 & 0.1850 & 0.3069 & 1
\end{array}\right] .
$$


TABLE 1: Channel models parameters.

\begin{tabular}{|c|c|c|c|c|c|}
\hline TAP index $K$ & Energy & Weibull fading factor $\beta$ & $P_{00, K}$ & $P_{11, K}$ & $P_{1}$ \\
\hline \multicolumn{6}{|c|}{ UIC } \\
\hline 1 & 0.756 & 2.49 & NA & 1.0000 & 1 \\
\hline 2 & 0.120 & 1.75 & 0.0769 & 0.9640 & 0.9625 \\
\hline 3 & 0.051 & 1.68 & 0.3103 & 0.8993 & 0.8732 \\
\hline 4 & 0.034 & 1.72 & 0.3280 & 0.8521 & 0.8199 \\
\hline 5 & 0.019 & 1.65 & 0.5217 & 0.7963 & 0.7017 \\
\hline 6 & 0.012 & 1.6 & 0.6429 & 0.7393 & 0.5764 \\
\hline 7 & 0.006 & 1.69 & 0.6734 & 0.6686 & 0.4971 \\
\hline \multicolumn{6}{|c|}{ UOC } \\
\hline 1 & 0.88 & 3.19 & NA & 1.0000 & 1.0000 \\
\hline 2 & 0.08 & 1.61 & 0.2717 & 0.9150 & 0.8956 \\
\hline 3 & 0.03 & 1.63 & 0.4401 & 0.8171 & 0.7538 \\
\hline 4 & 0.01 & 1.73 & 0.5571 & 0.7488 & 0.63825 \\
\hline \multicolumn{6}{|c|}{ Small city } \\
\hline 1 & 0.90 & 3.95 & NA & 1.0000 & 1.0000 \\
\hline 2 & 0.08 & 1.91 & 0.4839 & 0.9446 & 0.9034 \\
\hline 3 & 0.02 & 2.02 & 0.3452 & 0.7712 & 0.7383 \\
\hline \multicolumn{6}{|c|}{ OHT } \\
\hline 1 & 0.95 & 4.3 & NA & 1.0000 & 1.0000 \\
\hline 2 & 0.04 & 1.64 & 0.3625 & 0.8366 & 0.7960 \\
\hline 3 & 0.01 & 1.89 & 0.5999 & 0.6973 & 0.5696 \\
\hline \multicolumn{6}{|c|}{ OLT } \\
\hline 1 & 0.96 & 5.15 & NA & 1.0000 & 1.0000 \\
\hline 2 & 0.04 & 1.63 & 0.3836 & 0.8525 & 0.8073 \\
\hline
\end{tabular}

TABLE 2: Correlation matrix for UIC (lower/upper triangular part: amplitude/phase).

\begin{tabular}{lccccccc}
\hline$m, n$ & 1 & 2 & 3 & 4 & 5 & 6 \\
\hline 1 & 1.00 & 0.1328 & 0.2456 & 0.3106 & 0.2238 & 0.1092 \\
2 & 0.1989 & 1.00 & 0.2052 & 0.1478 & 0.0146 & 0.2508 & 0.1464 \\
3 & 0.0555 & 0.1477 & 1.00 & 0.0254 & 0.3209 & 0.2981 \\
4 & 0.0481 & 0.1495 & 0.2298 & 1.00 & 0.1591 & 0.0769 & 0.1460 \\
5 & 0.0977 & 0.0974 & 0.0106 & 0.2189 & 1.00 & 0.3258 \\
6 & 0.1074 & 0.2329 & 0.1368 & 0.2088 & 0.16 & 0.1471 & 1.00 \\
7 & 0.3504 & 0.1999 & 0.1496 & 0.1143 & 0 & 0.26 & 0.2059 \\
\hline
\end{tabular}

A commonly used method to evaluate the performance is the mean-square error (MSE) between the estimated and the desired data

$$
\mathrm{MSE}=\frac{1}{L} \sum_{l=1}^{L}\left(\widehat{Y}_{l}-Y_{l}\right)^{2}
$$

To evaluate the accuracy of the TDL based V2V channel model, we employ the UOC channel parameters to generate non-WSSUS channel model. The desired correlation coefficients matrix is in Table 3 , and the generated correlation coefficients matrix is $\rho_{\mathrm{Gen}}^{(W)}$ and $\rho_{\mathrm{Gen}}^{(U)}$. The MSE between the desired correlation coefficients matrix and the generated correlation coefficients matrix can be used to show the performance of the TDL based non-WSSUS V2V channel model. The MSE in matrix form is

$$
\begin{aligned}
\operatorname{MSE}^{(W)} & =\left[\begin{array}{cccc}
1 & 2.49 & 1.67 & 3.16 \\
2.49 & 1 & 2.75 & 1.73 \\
1.67 & 2.75 & 1 & 2.28 \\
3.16 & 1.73 & 2.28 & 1
\end{array}\right] \times 10^{-2}, \\
\mathbf{M S E}^{(U)} & =\left[\begin{array}{cccc}
1 & 1.42 & 2.55 & 1.30 \\
1.42 & 1 & 2.14 & 1.71 \\
2.55 & 2.14 & 1 & 0.92 \\
1.30 & 1.71 & 0.92 & 1
\end{array}\right] \times 10^{-2},
\end{aligned}
$$

where the matrix $\mathbf{M S E}^{(W)}$ is the MSE of the amplitude correlation coefficients matrix and the matrix $\mathbf{M S E}^{(U)}$ is the MSE of the phase correlation coefficients matrix. The MSE 
TABLE 3: Correlation matrix for UOC (lower/upper triangular part: amplitude/phase).

\begin{tabular}{lcccc}
\hline$m, n$ & 1 & 2 & 3 & 4 \\
\hline 1 & 1.00 & 0.2831 & 0.0243 & 0.1355 \\
2 & 0.6898 & 1.00 & 0.3046 & 0.1430 \\
3 & 0.6518 & 0.4922 & 1.00 & 0.2741 \\
4 & 0.5772 & 0.5142 & 0.8479 & 1.00 \\
\hline
\end{tabular}

TABLE 4: Correlation matrix for Small City (lower/upper triangular part: amplitude/phase).

\begin{tabular}{lccc}
\hline$m, n$ & 1 & 2 & 3 \\
\hline 1 & 1.00 & 0.3011 & 0.2056 \\
2 & 0.0338 & 1.00 & 0.1044 \\
3 & 0.6813 & 0.0684 & 1.00 \\
\hline
\end{tabular}

TABLE 5: Correlation matrix for OHT (lower/upper triangular part: amplitude/phase).

\begin{tabular}{lccc}
\hline$m, n$ & 1 & 2 & 3 \\
\hline 1 & 1.00 & 0.2152 & 0.0240 \\
2 & 0.5441 & 1.00 & 0.3026 \\
3 & 0.4157 & 0.1707 & 1.00 \\
\hline
\end{tabular}

TABLE 6: Correlation matrix for OLT (lower/upper triangular part: amplitude/phase).

\begin{tabular}{lcc}
\hline$m, n$ & 1 & 2 \\
\hline 1 & 1.00 & 0.1044 \\
2 & 0.1977 & 1.00 \\
\hline
\end{tabular}

specifies that the generated taps have a good approximation to the desired correlated taps.

The accuracy of the proposed channel model with respect to measurements is compared with the basis of the simulated power delay profiles (PDPs). In Figure 4, we compare the PDPs of the proposed channel model with measurements in scenarios: UIC, UOC, Small City, OHT, and OLT. The spread delay in UIC scenario is more severe than others, because the UIC scenario is antenna inside the car, which leads to more obstacles in channel propagation. In OLT scenario, which is low traffic density, the scattering and reflection caused by moving cars are less than other scenarios, the spread delay is short, and the energies are centered in the first tap. The simulation results of PDPs are consistent with the measurements, which validates that the proposed channel model can accurately represent the non-WSSUS properties of V2V channels.

Furthermore we evaluate the performances of the OFDM systems under the proposed channel model condition. In simulation, the data rate is $20 \mathrm{Mb} / \mathrm{s}$, the subcarrier number is $N=64$, the cyclic prefix number is $N_{\text {cp }}=16$, and channel bandwidth is under $10 \mathrm{MHz}$. The simulation of the TDL based non-WSSUS channel model in OFDM system is always compared on the basis of bit error ratio (BER).

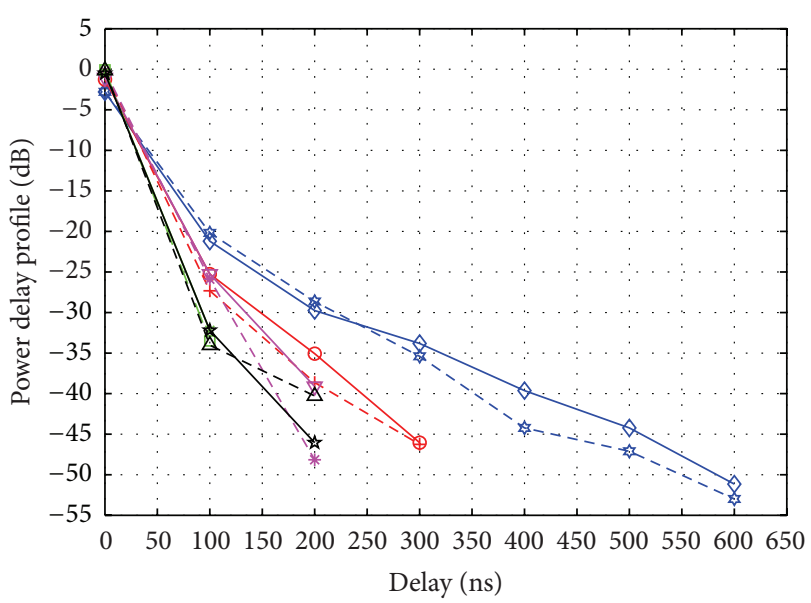

$$
\begin{array}{ll}
- \text { UIC measurement } & -*-\text { S simulation } \\
-\rightarrow-\text { UIC simulation } & -\star \text { OLT measurement } \\
- \text { UOC measurement } & - \text { - OLT simulation } \\
-+- \text { UOC simulation } & -\star \text { OHT measurement } \\
- \text { S measurement } & -\triangle-\text { OHT simulation }
\end{array}
$$

Figure 4: Power delay profile of the TDL based non-WSSUS V2V channel model in OFDM system (five scenarios: UIC, UOC, Small City, OLT, and OHT).

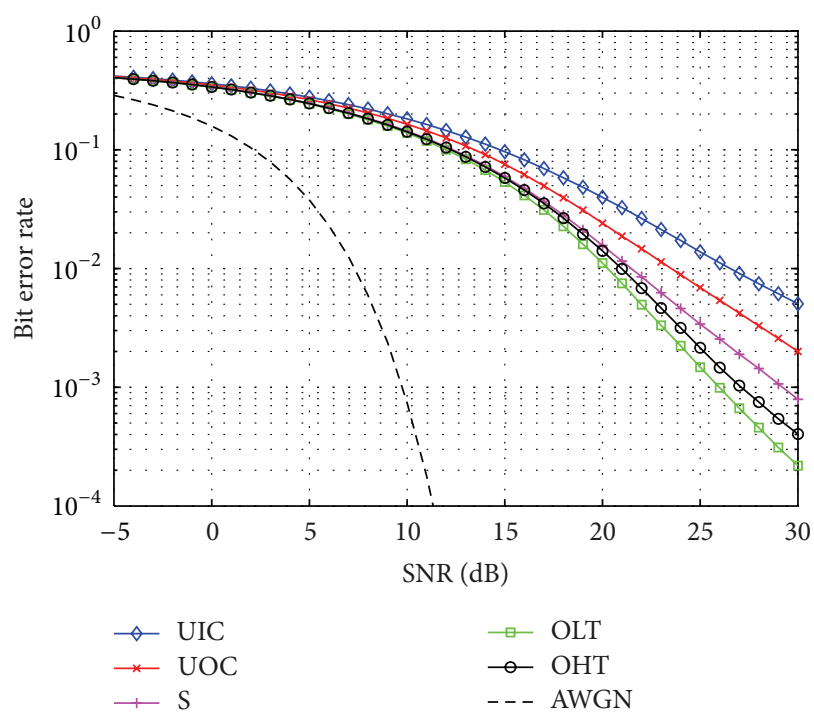

FIGURE 5: Bit error rate of the TDL based non-WSSUS V2V channel model in OFDM systems (five scenarios: UIC, UOC, Small City, OLT, and OHT).

Comparing the BER of simulating five scenarios and AWGN channels in Figure 5.

The presented results have been calculated with a SNR of -5 and $30 \mathrm{~dB}$; it is clear that OLT scenario channel condition is the best and UIC scenario channel condition is the worst. The reason is that OLT is open-area low traffic density scenario; the obstacles such as moving cars are less than other scenarios; UIC is antenna inside the car; the propagation paths are all through the transmitter and receiver cars, which causes severe fading and strong reflections. Due 
to the antenna inside the car, the channel condition of the UIC scenario presents a large spread delay and achieves the highest BER. The low traffic scenario presents a short delay spread and no further multipath contributions thus achieving a small BER.

\section{Conclusions}

In this paper, a TDL based channel model that characterized the non-WSSUS properties of $\mathrm{V} 2 \mathrm{~V}$ communications is proposed. The taps with different delays are correlated both in amplitude and phase. Utilizing the relationship between the correlation coefficients of complex Gaussian, Weibull, and Uniform RVs, the correlated taps are generated with arbitrary amplitude and phase correlation coefficients, fading parameters, and energy parameters. Using the MSE to evaluate the accuracy of the proposed channel model, the generated correlation coefficients are close to the desired correlation coefficients. The simulation results of the V2V channel PDPs show that the proposed channel model can simulate the V2V channel accurately and effectively.

\section{Acknowledgments}

This work is supported by the National Natural Science Foundation of China under Grant 61222105, the Fundamental Research Funds for the Central Universities under Grant 2013YJS005, Beijing Municipal Natural Science Foundation under Grant 4112048, Project of State Key Lab under Grant no. RCS2012ZT013, key Project for Railway Ministry of China under Grant 2012X008-A, interdiscipline cooperation projects of the New-Star of Science and Technology supported by Beijing Metropolis under Grant no. xxhz201201, and the Key Grant Project of Chinese Ministry of Education under Grant 313006.

\section{References}

[1] ITS Project, 2007, http://www.its.dot.gov/index.htm.

[2] IEEE P802.11p/D2.01, "Standard for wireless local area networks providing wireless communication," in Proceedings of the 5th European Congress Exhibition Intelligent Transportation System Services, Hannover, Germany, June 2005.

[3] J. Wang, S. Jia, and J. Song, "Signal vector based detection scheme for spatial modulation," IEEE Communications Letters, vol. 16, no. 1, pp. 19-21, 2012.

[4] P. A. Bello, "Characterization of randomly time-variant linear channels," IEEE Transactions on Communications, vol. 11, no. 4, pp. 360-393, 1963.

[5] C.-X. Wang, X. Cheng, and D. Laurenson, "Vehicle-to-vehicle channel modeling and measurements: recent advances and future challenges," IEEE Communications Magazine, vol. 47, no. 11, pp. 96-103, 2009.

[6] J. Maurer, T. Fügen, T. Schäfer, and W. Wiesbeck, "A new intervehicle communications (IVC) channel model," in Proceedings of the IEEE 60th Vehicular Technology Conference (VTC '04), pp. 9-13, September 2004.
[7] J. Maurer, T. Fgen, M. Porebska, T. Zwick, and W. Wisebeck, "A ray-optical channel model for mobile to mobile communications," in Proceedings of the 4th MCM COST, vol. 2100, pp. $6-8,2008$.

[8] J. Wang, S. Jia, and J. Song, "Generalised spatial modulation system with multiple active transmit antennas and low complexity detection scheme," IEEE Transactions on Wireless Communications, vol. 11, no. 4, pp. 1605-1615, 2012.

[9] A. S. Akki and F. Haber, "A statistic model for mobile-to-mobile land communication channel," IEEE Transactions on Vehicular Technology, vol. 35, no. 1, pp. 2-7, 1986.

[10] X. Cheng, C.-X. Wang, D. I. Laurenson, S. Salous, and A. V. Vasilakos, "An adaptive geometry-based stochastic model for non-isotropic MIMO mobile-to-mobile channels," IEEE Transactions on Wireless Communications, vol. 8, no. 9, pp. 4824-4835, 2009.

[11] A. G. Zajić and G. L. Stüber, "Three-dimensional modeling and simulation of wideband MIMO mobile-to-mobile channels," IEEE Transactions on Wireless Communications, vol. 8, no. 3, pp. 1260-1275, 2009.

[12] J. Karedal, F. Tufvesson, N. Czink et al., "A geometry-based stochastic MIMO model for vehicle-to-vehicle communications," IEEE Transactions on Wireless Communications, vol. 8, no. 7, pp. 3646-3657, 2009.

[13] X. Cheng, Q. Yao, C. X. Wang et al., “An improved parameter computation method for a MIMO V2V rayleigh fading channel simulator under non-isotropic scattering environments," IEEE Communications Letters, vol. 17, no. 2, pp. 265-2268, 2013.

[14] X. Cheng, C. X. Wang, B. Ai, and H. Aggoune, "Envelope level crossing rate and average fade duration of non-isotropic vehicle-to-vehicle ricean fading channels," IEEE Transactions on Intelligent Transportation, no. 99, pp. 1-11.

[15] X. Cheng, Q. Yao, M. Wen, C. X. Wang, L. Song, and B. Jiao, "Wideband channel modeling and ICI cancellation for vehicleto-vehicle communication systems," IEEE Journal on Selected Areas in Communications, vol. 31, no. 9.

[16] G. Acosta and M. A. Ingram, "Model development for the wideband expressway vehicle-to-vehicle $2.4 \mathrm{GHz}$ channel," in Proceedings of the IEEE Wireless Communications and Networking Conference (WCNC '06), pp. 1283-1288, April 2006.

[17] G. Acosta-Marum and M. A. Ingram, "Six time- and frequencyselective empirical channel models for vehicular wireless LANs," IEEE Vehicular Technology Magazine, vol. 2, no. 4, pp. 4-11, 2007.

[18] I. Sen and D. W. Matolak, "Vehicle-vehicle channel models for the 5-GHz band," IEEE Transactions on Intelligent Transportation Systems, vol. 9, no. 2, pp. 235-245, 2008.

[19] E. Kreyszig, Advanced Engineering Mathematics, Wiley, New York, NY, USA, 8th edition, 1988.

[20] J. Fühl, J.-P. Rossi, and E. Bonek, "High-resolution 3-d direction-of-arrival determination for urban mobile radio," IEEE Transactions on Antennas and Propagation, vol. 45, no. 4, pp. 672-682, 1997.

[21] N. C. Sagias and G. K. Karagiannidis, "Gaussian class multivariate Weibull distributions: theory and applications in fading channels," IEEE Transactions on Information Theory, vol. 51, no. 10, pp. 3608-3619, 2005.

[22] N. L. Johnson and S. Kotz, Distributions in Statistics: Continuous Multivariate Distributions, Wiley, New York, NY, USA, 1972. 

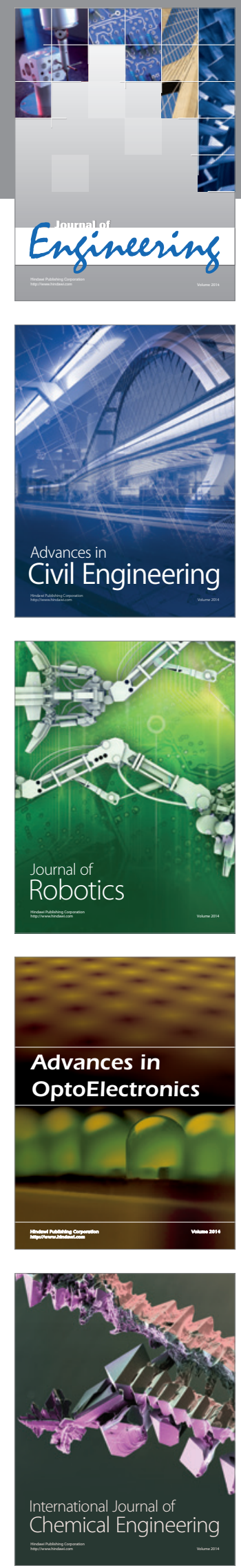

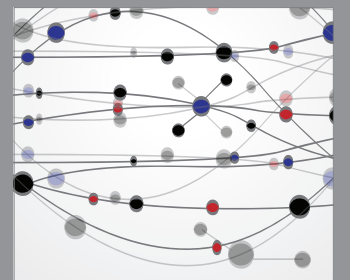

The Scientific World Journal
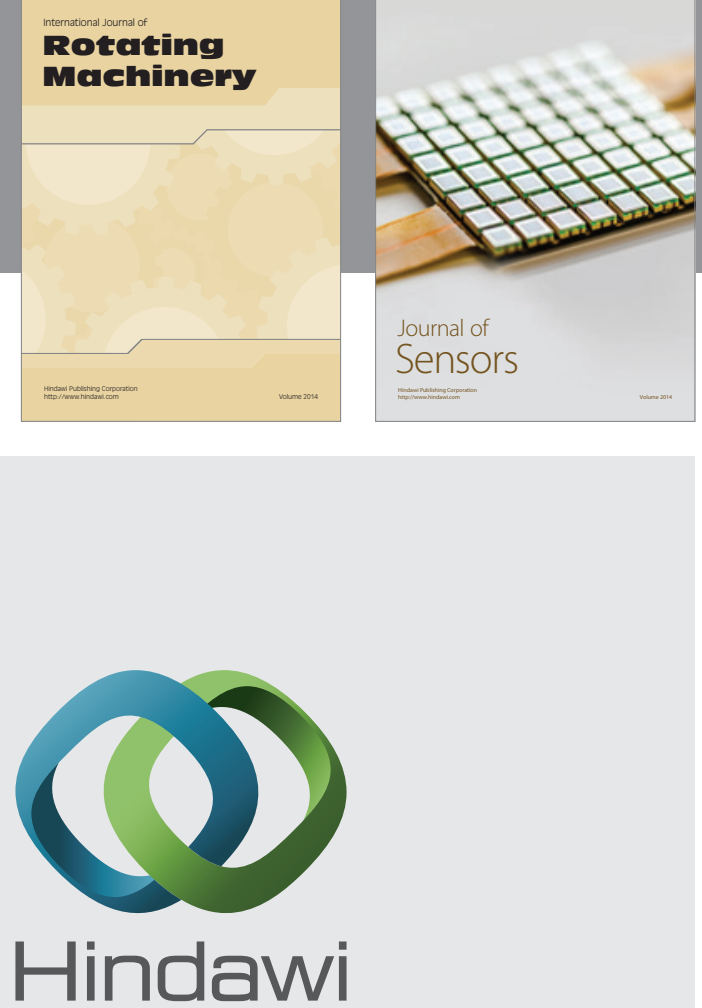

Submit your manuscripts at http://www.hindawi.com
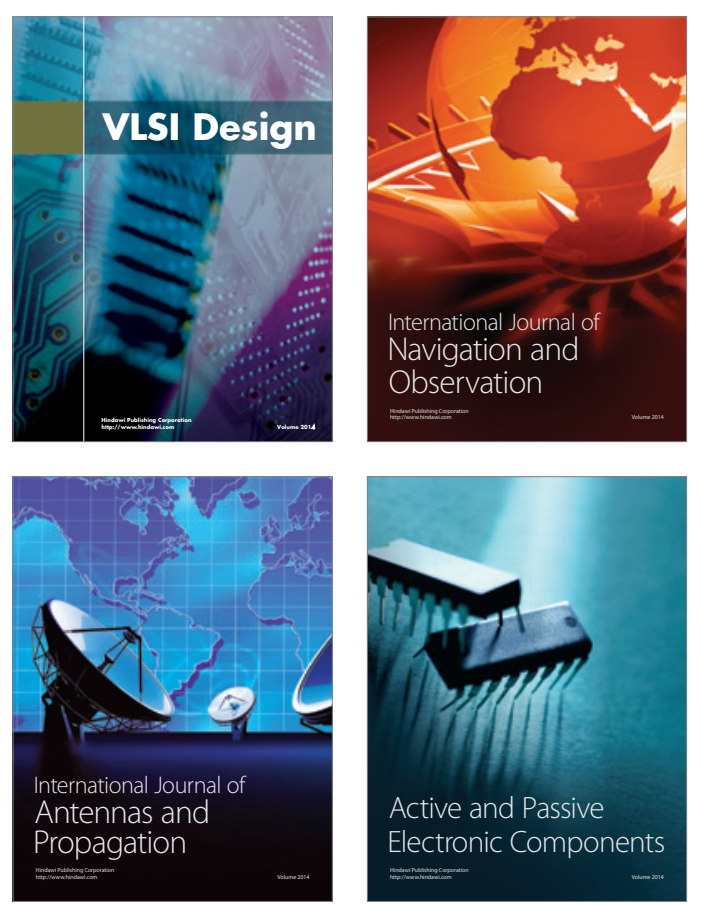
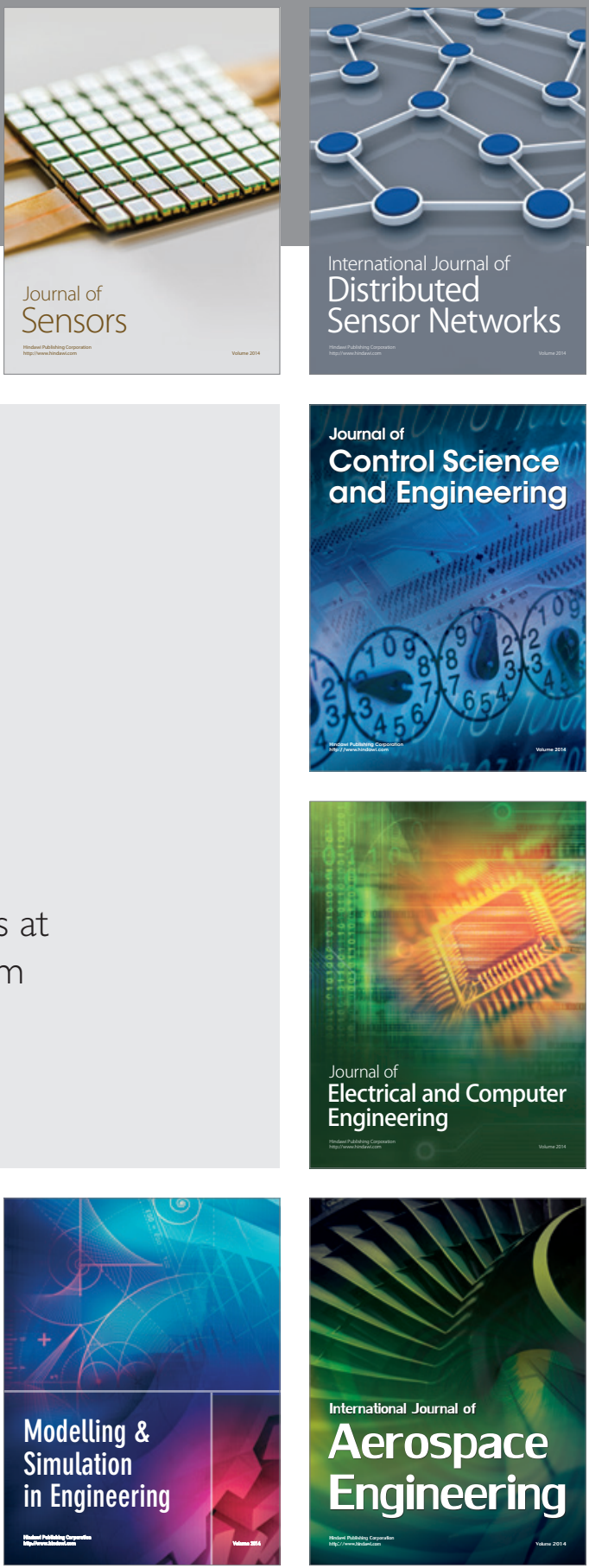

Journal of

Control Science

and Engineering
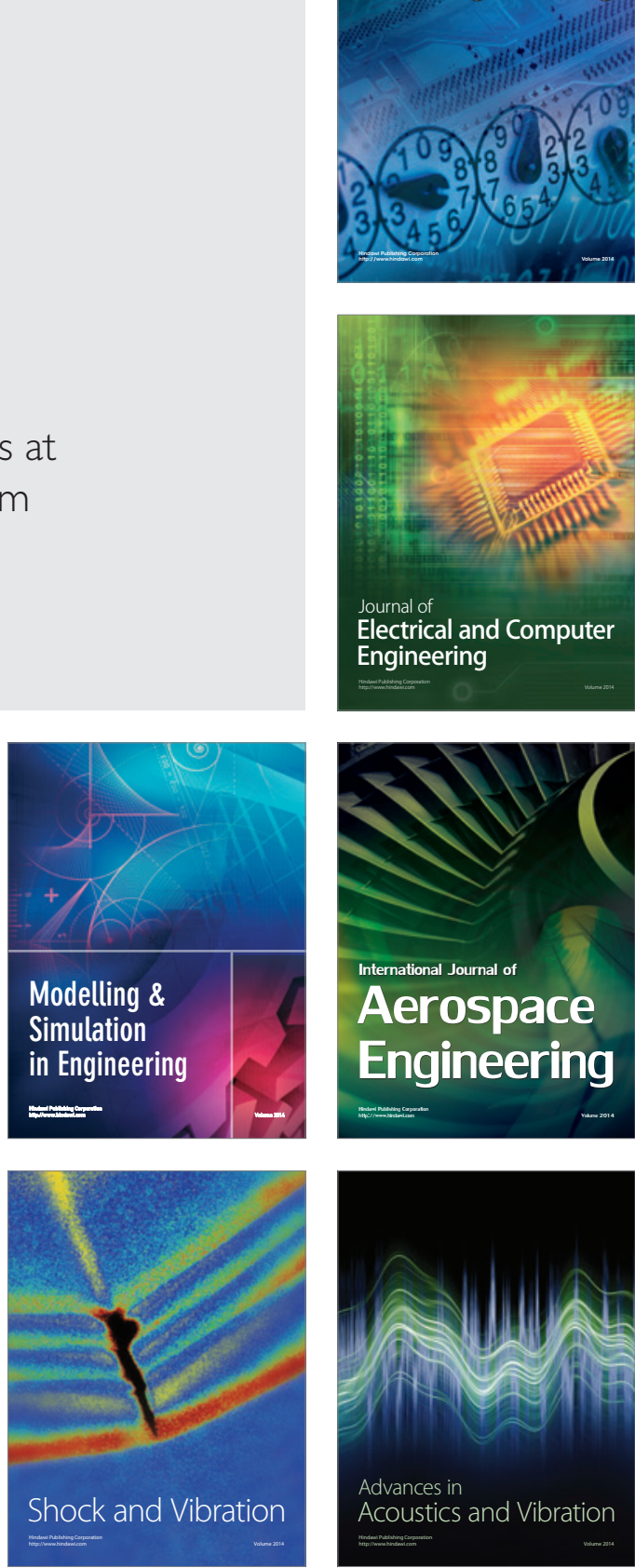